\title{
Analysis of Current Distributions and Radar Cross Sections of Line Source Scattering from Impedance Strip by Fractional Derivative Method Kamil Karaçuha ${ }^{1, *}$, E. I. Veliyev ${ }^{1,2}$, Vasil Tabatadze $^{1,3}$, Ertuğrul Karaçuha ${ }^{1}$
}

\author{
(1) Informatics Institute of Istanbul Technical University, Istanbul Turkey \\ ${ }^{(2)}$ National University of 'Kharkiv Polytechnic Institute', Kharkiv, Ukraine \\ ${ }^{(3)}$ Tbilisi State University, Tbilisi, Georgia \\ *E-mail: karacuha17@itu.edu.tr
}

\begin{abstract}
In this paper, we have studied the analysis of current distributions and radar cross sections of the line source scattering from an impedance strip. The problem was solved with the fractional derivative method previously. Here, the specific case of the fractional derivative method is investigated. The problem under consideration on the basis of various methods is studied well, however, they are mainly done by numerical methods. The fractional derivative method allows one to obtain an analytical solution in a specific situation. This method allows to obtain analytical solution of impedance strip for a special case which is fractional order $v$ is equal to 0.5 . When fractional order is 0.5 , there is an analytical solution which is explained in this paper and current distribution, radar cross section and near field patterns are derived. Here, as a first time, the current distribution, the bi-static radar cross section and the near field for the upper and the lower part of the strip are studied.
\end{abstract}

\section{Introduction}

The application of the fractional calculus such as fractional operators, transforms or the fractionalization of some known operators are studied well previously [1 - 4]. This allows us to describe intermediate states for different physical phenomena. In the electromagnetic theory, fractional operators firstly are used by N. Engheta and the study is called as "fractional paradigm in electromagnetic theory" [3]. Here, our purpose is to use the fractional derivative method (FDM) which is firstly proposed by E. I. Veliyev for solving diffraction problems.

Here, the two-dimensional infinitely thin surface strip is studied. As a source term, line source is introduced. Previously, the general formulation and field distributions for the upper space of the strip, the monostatic radar cross section were studied $[5,6]$ whereas, in this paper, the current distribution, the upper and the lower part of the scattered and the total field and bi-static radar cross sections for the line source with an arbitrary distance and angle are studied for the fractional order (FO) $v, 0.5$. In order to solve this specific diffraction problem, new fractional boundary condition (FBC) on the strip is introduced. FBC covers both perfect electric conducting (PEC) and the perfect magnetic conducting (PMC) cases with the change in the FO [5].

The fractional derivative is denoted as $D_{y}^{v}$ which means the derivative with respect to $\mathrm{y}$ variable and the order of derivative is $v$. Here, we will use the Riemann - Liouville definition of the derivative which is valid for the fractional order between $(0,1)$ [5]. Due to knowing that the derivative is a linear operator, derivatives with other fractional orders can be found a combination of integer derivative with the fractional derivative.

$$
\mathfrak{D}_{y}^{v} f(y)=\frac{1}{\Gamma(1-v)} \frac{d}{d y} \int_{-\infty}^{y} \frac{f(t)}{(y-t)^{v}} d t
$$

where, the fractional order $v \epsilon(0,1)$ and $\Gamma(v)$ is the Gamma Function. In order to define the fractional derivative fully, (2) is given.

$$
\mathfrak{D}_{\mathrm{y}}^{v} \mathrm{f}(\mathrm{y})=\left\{\begin{array}{c}
f(y), \quad v=0 \\
\mathfrak{D}_{\mathrm{y}}^{v} \mathrm{f}(\mathrm{y}), \quad 0<v<1 \\
f^{\prime}(y), \quad v=1
\end{array}\right.
$$

After the definition, it is needed to express the fractional boundary condition, mathematically which is the boundary condition can cover both known Dirichlet and Neumann boundary conditions (FBC) [5]. For our problem which is two-dimensional strip located at $y=0$ with some finite width, FBC can be found as

$$
\left.\mathfrak{D}_{k y}^{v} E_{z}(x, y)\right|_{y= \pm 0}=0
$$

where, $E_{z}$ is the total electric field component along z-axis on the surface of the strip. In (3), for different $v$ values, the fractional boundary condition corresponds to different 
materials. If the fractional order $v$ is equal to 0 , the boundary is $\mathrm{PEC}$, on the other hand, if $v=1$, the boundary becomes PMC.

\section{Formulation of the Problem}

The problem is to investigate what is the current distribution on the surface of the strip, the near electric field and the bistatic radar cross section which has not studied yet. A twodimensional strip of width $2 \mathrm{a}$ on the plane $\mathrm{y}=0$ is located. The strip along the z-axis is infinite. The source of the cylindrical wave $\vec{J}_{e}=\vec{z} J_{e} \delta\left(x-x_{o}\right) \delta\left(y-y_{o}\right)$ is located at the point $\left(x_{o}, y_{o}\right)$ as shown in Fig. 1. The time dependency is given as $e^{-i \omega t}$ and throughout the problem, it will be omitted.

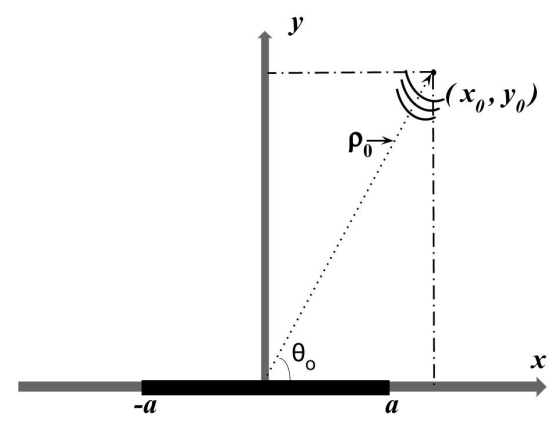

Figure 1: The geometry of the problem

Let us consider the case of an E-polarized wave, i.e. $\vec{E}_{z}^{i}\left(0,0, E_{z}\right), \vec{H}_{z}^{i}\left(H_{x}, H_{y}, 0\right)$. In this case, the source field has the form [7]

$$
\vec{E}_{z}^{i}(x, y)=-\overrightarrow{J_{e}} \frac{\eta_{0} k}{4} H_{0}^{(1)}\left(k \sqrt{\left(x-x_{o}\right)^{2}+\left(y-y_{o}\right)^{2}}\right)
$$

Here, $H_{0}^{(1)}(k \mathrm{x})$ is the Hankel function of the first kind and zero order, $\eta_{0}$ is the impedance of free space, and $k=\frac{2 \pi}{\lambda}$ is the wave number. The total electric field can be represented as a superposition of the fields below.

$$
\vec{E}_{z}=\vec{E}_{z}^{i}+\vec{E}_{z}^{s}
$$

Our purpose is to find the scattered field which must satisfy FBC. The scattered field can be found as (6).

$$
E_{z}^{s}(x, y)=\int_{-\infty}^{\infty} f^{1-v}\left(x^{\prime}\right) G^{v}\left(x-x^{\prime}, y\right) d x^{\prime}
$$

Here, $f^{1-v}\left(x^{\prime}\right)$ is an unknown function, which we will call the fractional density of the potential, and the fractional Green's function $G^{v}(x)$ has the form [5-8]. We can use the spectral representation of the Hankel Function.

$$
G^{v}\left(x-x^{\prime}, y\right)=-\frac{i}{4} \mathfrak{D}_{k y}^{v} H_{0}^{(1)}\left(k \sqrt{\left(x-x^{\prime}\right)^{2}+y^{2}}\right)
$$

where,

$$
\begin{aligned}
& H_{0}^{(1)}\left(k \sqrt{\left(x-x^{\prime}\right)^{2}+y^{2}} \int_{\infty}\right. \\
& =\frac{1}{\pi} \int_{-\infty}^{\infty i k\left(\left(x-x^{\prime}\right) \alpha+|y| \sqrt{1-\alpha^{2}}\right)} \frac{d \alpha}{\sqrt{1-\alpha^{2}}}
\end{aligned}
$$

Here, $\operatorname{Im}\left\{\sqrt{1-\alpha^{2}}>0\right\}$ is assumed. $\operatorname{Im}\{f(\alpha)\}$ means imaginary part of the function $f(\alpha)$.

Using (6) and (7), the scattered field can be found as

$$
\begin{aligned}
& E_{z}^{S}(x, y) \\
= & -i \frac{e^{i \frac{\pi}{2} v}}{4 \pi} \int_{-\infty}^{\infty} F^{1-v}(\alpha) e^{i k\left[\alpha x+y \sqrt{1-\alpha^{2}}\right]}\left(1-\alpha^{2}\right)^{\frac{v-1}{2}} d \alpha
\end{aligned}
$$

where,

$$
\begin{aligned}
& F^{1-v}(\alpha)=\int_{-1}^{1} \tilde{f}^{1-v}(\xi) e^{-i \varepsilon \alpha \xi} d \xi, \tilde{f}^{1-v}(\xi)=a f^{1-v}(a \xi) \\
& \varepsilon=k a, \quad \xi=\frac{x}{a}, \quad \tilde{f}^{1-v}(\xi)=\frac{\varepsilon}{2 \pi} \int_{-\infty}^{\infty} F^{1-v}(\alpha) e^{i \varepsilon \alpha \xi} d \alpha
\end{aligned}
$$

Here, $F^{1-v}(\alpha)$ is the Fourier transform of $\tilde{f}^{1-v}(\xi)$ which is the normalized current density on the strip.

After, finding the scattered field expression, the FBC given in (9) needs to be applied to the total field.

$$
\left.\mathfrak{D}_{k y}^{v} E_{z}(x, y)\right|_{y= \pm 0}=0
$$

where, $x,-a<x<a$ and $v$ is a fractional order (FO).

After applying $\mathrm{FBC}$, we obtain the following integral equation (IE) given in (10).

$$
\begin{aligned}
& -i \frac{e^{i \pi v}}{4 \pi} \int_{-\infty}^{\infty} F^{1-v}(\alpha) e^{i k \alpha x}\left(1-\alpha^{2}\right)^{v-1 / 2} d \alpha \\
& \quad=J_{e} \frac{\eta_{0} k}{4 \pi} e^{i \frac{\pi}{2} v} \int_{-\infty}^{\infty} e^{i k\left(\left(x-x_{0}\right) \alpha-y_{0} \sqrt{1-\alpha^{2}}\right)}\left(1-\alpha^{2}\right)^{\frac{v-1}{2}} d \alpha
\end{aligned}
$$

For the general solution, both sides of (10) with $e^{-i k x \beta}$ needs to be multiplied and needs to be taken the integral from $-\mathrm{a}$ to $+\mathrm{a}$ with respect to $\mathrm{x}$ variable. Then, (10) becomes as (11) given below.

$\int_{-\infty}^{\infty} F^{1-v}(\alpha) \frac{\sin \varepsilon(\alpha-\beta)}{\alpha-\beta}\left(1-\alpha^{2}\right)^{v-\frac{1}{2}} d \alpha=$ 


$$
-4 i B \pi e^{-i \frac{\pi}{2} \nu} \int_{-\infty}^{\infty} e^{i k\left[-x_{0} \alpha+y_{0} \sqrt{1-\alpha^{2}}\right]} \frac{\sin \varepsilon(\alpha-\beta)}{\alpha-\beta}\left(1-\alpha^{2}\right)^{\frac{\nu-1}{2}} d \alpha
$$

where, $B=-J_{e} \frac{\eta_{0} k}{4 \pi}$

For the fractional order $v$ is equal to 0.5 , the normalized current density can be found directly from (10) and the current density becomes as (12) by using the inverse Fourier transform.

$$
\begin{aligned}
& \tilde{f}^{0.5}(\xi)= \\
& \text { and, } \\
& \quad-i 2 \varepsilon B e^{\mp i \frac{\pi}{4}} \int_{-\infty}^{\infty} e^{i\left[\left(\varepsilon \alpha \xi-k x_{0} \alpha\right)+k y_{0} \sqrt{1-\alpha^{2}}\right]}\left(1-\alpha^{2}\right)^{-\frac{1}{4}} d \alpha
\end{aligned}
$$

$$
\begin{aligned}
& F^{0.5}(\alpha)= \\
& -i 4 B e^{\mp i \frac{\pi}{4}} \int_{-\infty}^{\infty} \frac{\sin \varepsilon(\beta-\alpha)}{(\beta-\alpha)} e^{i\left[\left(-k x_{0} \beta\right)+k y_{0} \sqrt{1-\beta^{2}}\right]}\left(1-\beta^{2}\right)^{-\frac{1}{4}} d \beta
\end{aligned}
$$

Equations (12) are the special case where the normalized current density $\tilde{f}^{0.5}$ and the Fourier transform of the current density $F^{0.5}$ have been found analytically.

It is better to examine the cylindrical line source in the far field with an arbitrary angle. In the cylindrical coordinate system $(\rho, \varphi)$, x can be written as $\rho \cos \varphi$ and y can be written as $\rho \sin \varphi$. Then, by using the steepest descent method and the coordinate change [6], for the large values of $k \rho_{0}$, the part of (12) related to the Fourier transform of the current density becomes as

$$
\begin{aligned}
& F^{0.5}(\alpha)= \\
& \quad-i 4 B e^{\mp i \frac{\pi}{4}} \sqrt{\frac{2 \pi}{k \rho_{0}}} \sqrt{\sin \theta_{0}} \frac{\sin \left(\epsilon\left(\cos \theta_{0}+\alpha\right)\right)}{\left(\cos \theta_{0}+\alpha\right)} e^{i k \rho_{0}-i \pi / 4}
\end{aligned}
$$

If $\mathrm{k} \rho \rightarrow \infty$, we can use the method of stationary phase to derive the expression for the far-field $E_{z}^{s}(\rho, \varphi)$ as follows

$$
E_{z}^{s}(\rho, \varphi)=A(k \rho) \Phi^{v}(\varphi) \text { while } \mathrm{k} \rho \rightarrow \infty
$$

where,

$$
\begin{gathered}
A(k \rho)=\sqrt{\frac{2}{\pi k \rho}} e^{i k \rho-i \pi / 4} \\
\Phi^{0.5}(\varphi)=-\frac{i}{4}( \pm i)^{v} F^{0.5}(\cos \varphi) \sqrt{\sin \varphi}
\end{gathered}
$$

For (15), ( + ) sign is taken into account if $0<\varphi<\pi$, ), and (-) sign is taken into account if $\pi<\varphi<2 \pi$. The function $\Phi^{v}(\varphi)$ stands for the radiation pattern (RP) of the scattered field. As given in (15), the far field expression is found analytically when the fractional order $v$, is equal to 0.5 .
As shown in $[9,10]$, the fractional order is related to the impedance. The relation between the fractional order $v$ and the impedance $\eta_{v}$ can be derived for the normal incidence plane wave as shown in (16)

$$
v=\frac{1}{i \pi} \ln \frac{1-\eta_{v}}{1+\eta_{v}}, \quad \eta_{v}=\frac{1}{i} \tan \left(\frac{\pi v}{2}\right)
$$

Here, we need to note that, this result is for the plane wave scattering, on the other hand, for the large value of $k \rho_{0}$ (see Fig. 1), it can be approximated as a plane wave and also in this article, and the approximation approach is given in (13) and (15). For the values of the fractional order $0<v<1$, $\eta_{v}$ is always pure imaginary [5].

The value $v=0$ corresponds to the impedance $\eta_{v}=0$ (PEC) and $v=1$ corresponds to $\eta_{v}=-i \infty$ (PMC). For the intermediate values $0<v<1$ the impedance has pure imaginary values between 0 and $-i \infty$. For a special case $\left(k \rho_{0} \rightarrow \infty\right)$, when $v=0.5$, impedance $\eta_{v}$ becomes $-i$ by using (16).

In order to understand the scattering phenomena, the Radar Cross Sections (RCS) also need to be investigated. Previously, the monostatic radar cross sections have been studied. Here both Bi-static and mono-static radar cross sections are studied. The formula for the bi-static RCS $\frac{\sigma_{2 \mathrm{~d}}}{\lambda}$ and the mono-static RCS $\sigma_{2 \mathrm{~d}}$ is derived from the expression for the RP $\Phi^{v}$ as given in (15). In order to calculate radar cross sections, (17) is used for the bi-static and the monostatic radar cross section, respectively [7].

$$
\frac{\sigma_{2 \mathrm{~d}}}{\lambda}(\varphi)=\frac{2}{\pi}|\Phi(\varphi)|^{2} ; \sigma_{2 \mathrm{~d}}(\text { monostatic })=\frac{\sigma_{2 \mathrm{~d}}}{\lambda}\left(\theta_{o}\right)
$$

\section{Numerical Analysis}

In order to discretize and solve IE (11) for any arbitrary fractional order, the current density can be given as following $[9,10]$.

$$
\tilde{f}^{1-v}(\xi)=\left(1-\xi^{2}\right)^{v-\frac{1}{2}} \sum_{n=0}^{\infty} f_{n}^{v} \frac{C_{n}^{v}(\xi)}{v}
$$

where, $C_{n}^{\nu}(\xi)$ denotes Gegenbauer polynomials which satisfy the boundary condition, and $f_{n}^{v}$ is the unknown coefficients. The current density needs to satisfy (19) at the edges [11].

$$
\tilde{f}^{1-v}(\xi)=O\left(\left(1-\xi^{2}\right)^{v-\frac{1}{2}}\right), \xi \rightarrow \pm 1
$$

The Fourier Transform of (18), in (20), $F^{1-v}(\alpha)$ can be found $[9,12]$. 


$$
F^{1-v}(\alpha)=\frac{2 \pi}{\Gamma(v+1)} \sum_{n=0}^{\infty}(-i)^{n} f_{n}^{v} \beta_{n}^{v} \frac{\mathcal{J}_{n+v}(\varepsilon \alpha)}{(2 \varepsilon \alpha)^{v}}
$$

Here, $\beta_{n}^{v}=\Gamma(\mathrm{n}+2 v) / \Gamma(\mathrm{n}+1)$ and $\mathcal{J}_{n+v}(\varepsilon \alpha)$ are the Bessel functions. Putting (20) into IE (10), we obtain a system of linear algebraic equations (SLAE) for determining the unknown coefficients $f_{n}^{v}$. SLAE has the form given in (21).

$$
\sum_{n=0}^{\infty}(-i)^{n} f_{n}^{v} \beta_{n}^{v} C_{m n}^{v}=\gamma_{m}^{v}
$$

Here,

$$
C_{m n}^{v}=\int_{-\infty}^{\infty} \mathcal{J}_{n+v}(\varepsilon \alpha) \mathcal{J}_{m+v}(\varepsilon \alpha)\left(1-\alpha^{2}\right)^{v-\frac{1}{2}} \frac{d \alpha}{\alpha^{2 v}}
$$

and

$$
\gamma_{m}^{v}=\int_{-\infty}^{\infty} \frac{\mathcal{J}_{m+\nu}(\varepsilon \alpha)}{\alpha^{v}} e^{i\left[-k x_{0} \alpha+k y_{0} \sqrt{1-\alpha^{2}}\right]}\left(1-\alpha^{2}\right)^{\frac{\nu-1}{2}} d \alpha
$$

where, $\Omega=\frac{i}{2 \pi} \Gamma(v+1) e^{-i \frac{\pi}{2} v}$

\section{Numerical Results}

In previous sections, the analytical and the numerical analysis have been studied. Here, for the fractional order $v=0.5$, the near field scattered and the total electric field given in Fig. 2-7. Then, the bi-static radar cross section (RCS) are investigated for the different source locations, angles and $\epsilon$. The results for bi-static RCS are given in Fig. 8 and 9. For bi-static RCS results, (17) is utilized. In Fig. 10 -12 , the normalized current densities for different angles of the incidence are shown. For these results, (18) is taken into account. As the purpose of this paper, the fractional order $v$ for all figures, is 0.5 . In all calculations, $B=-J_{e} \frac{\eta_{0} k}{4 \pi}$ used in (11) is assumed to be 1 .

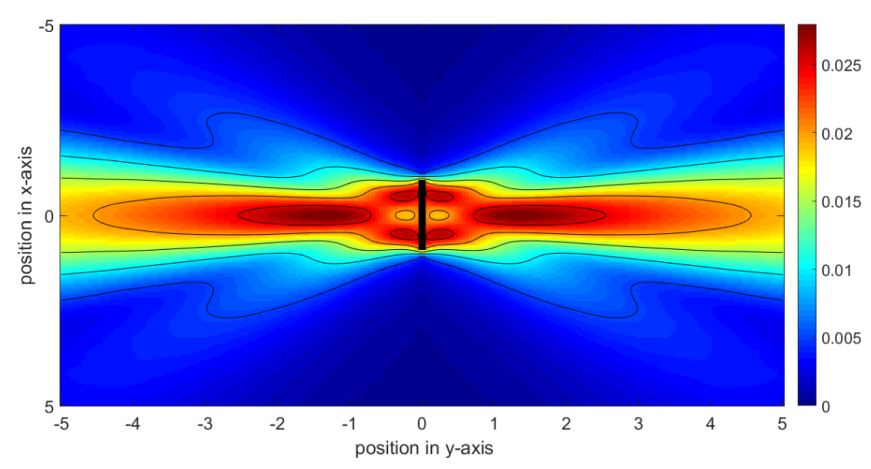

Figure 2: The Amplitude of the Scattered Electric Field $\vec{E}_{z}^{s}$ for $a=1, \epsilon=2 \pi, x_{0}=0$ and $y_{0}=2 \pi$

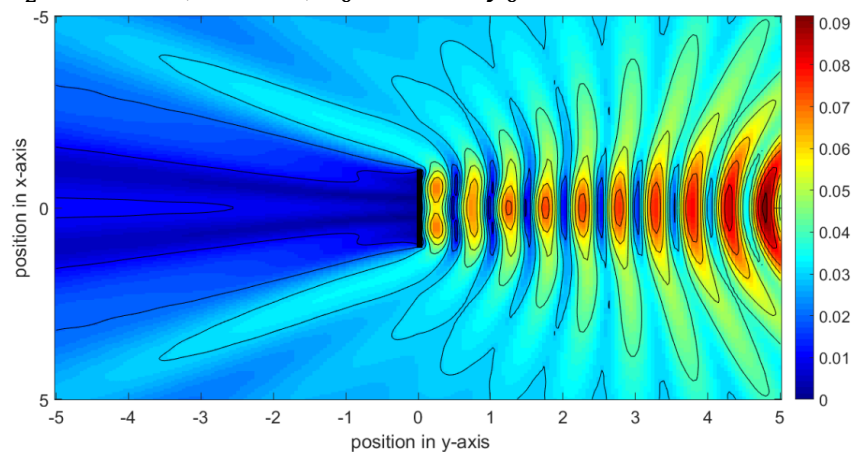

Figure 3: The Amplitude of the Total Electric Field $\vec{E}_{z}^{i}$ for $a=1, \epsilon=2 \pi, x_{0}=0$ and $y_{0}=2 \pi$

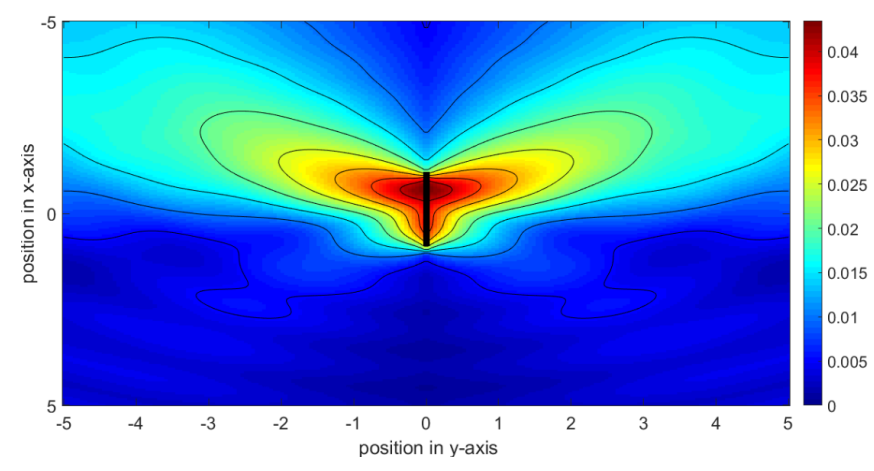

Figure 4: The Amplitude of the Scattered Electric Field $\vec{E}_{z}^{s}$ for $a=1, \epsilon=\pi, x_{0}=2 \pi$ and $y_{0}=2 \pi$

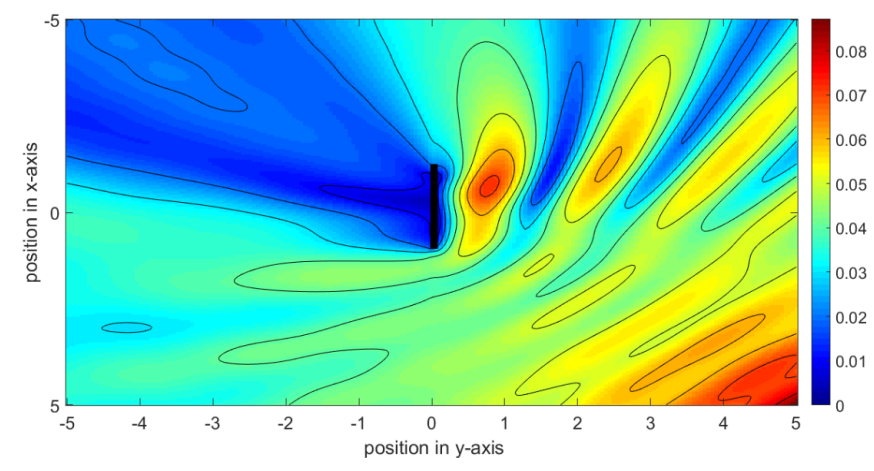

Figure 5: The Amplitude of the Total Electric Field $\vec{E}_{z}^{i}$ for $a=1, \epsilon=\pi, x_{0}=2 \pi$ and $y_{0}=2 \pi$ 


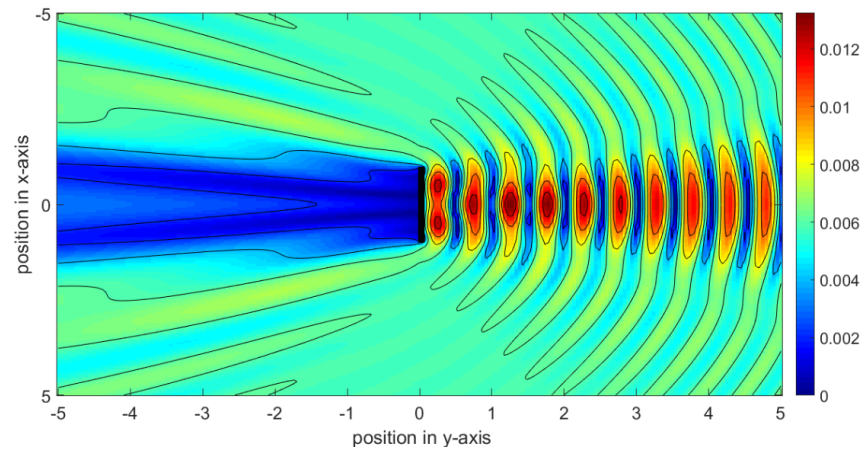

Figure 6: The Amplitude of the Total Electric Field $\vec{E}_{z}^{i}$ for $a=1, \epsilon=2 \pi, x_{0}=0$ and $y_{0}=6 \pi$

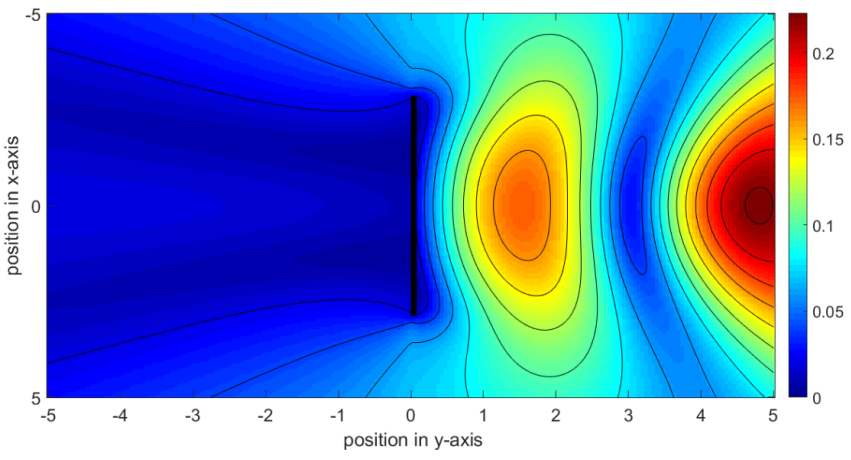

Figure 7: The Amplitude of the Total Electric Field $\vec{E}_{z}^{i}$ for $a=3, \epsilon=2 \pi, x_{0}=0$ and $y_{0}=2 \pi$

In Fig. 8 and 9, Bi-static RCS with different $\epsilon=k a$ values are shown. The location of the source line and the angle with respect to the strip are given in order. 1 is responsible for $\theta_{0}=90^{\circ}, x_{0}=0, y_{0}=\epsilon .2$ is responsible for $\theta_{0}=90^{\circ}$, $x_{0}=0, y_{0}=20 \epsilon .3$ is responsible for $\theta_{0}=45^{0}, x_{0}=3 \epsilon$, $y_{0}=3 \epsilon$ and 4 is responsible for $\theta_{0}=60^{\circ}, x_{0}=3 \epsilon, y_{0}=$ $3 \sqrt{3} \epsilon$

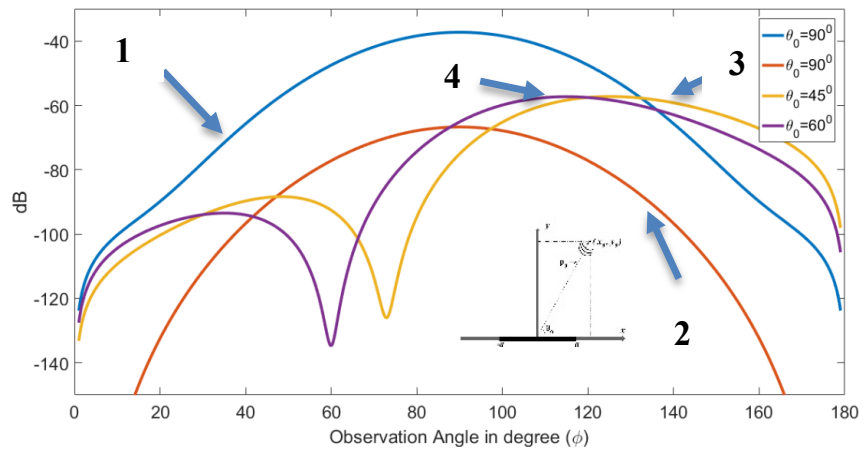

Figure 8: Bi-static Radar Cross Section for $\epsilon=\pi$

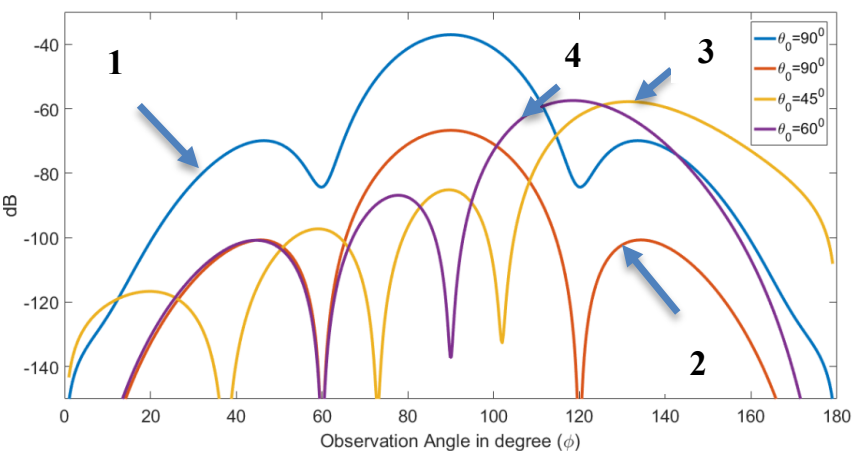

Figure 9: Bi-static Radar Cross Section for $\epsilon=2 \pi$

In Fig. 10, 11 and 12, the normalized current density are shown for the different $\epsilon$ and the source position $\rho_{0}$, The location of the source line and the angle with respect to strip are given in order. Arrows 1,2 and 3 are responsible for $\theta_{0}=90^{\circ}, \theta_{0}=40^{\circ}, \theta_{0}=60^{\circ}$, respectively.

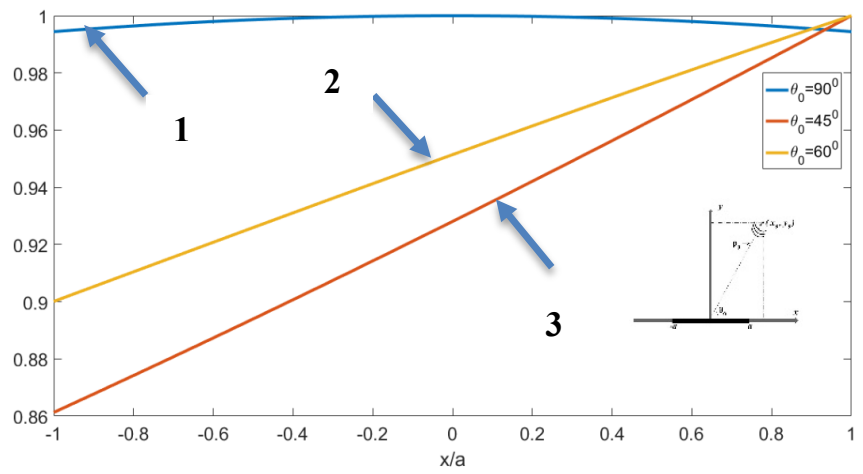

Figure 10: The normalized Fractional Current Density $\left|\tilde{f}^{1-v}(\xi)\right|$ for $\epsilon=\pi$ and $\rho_{0}=3$

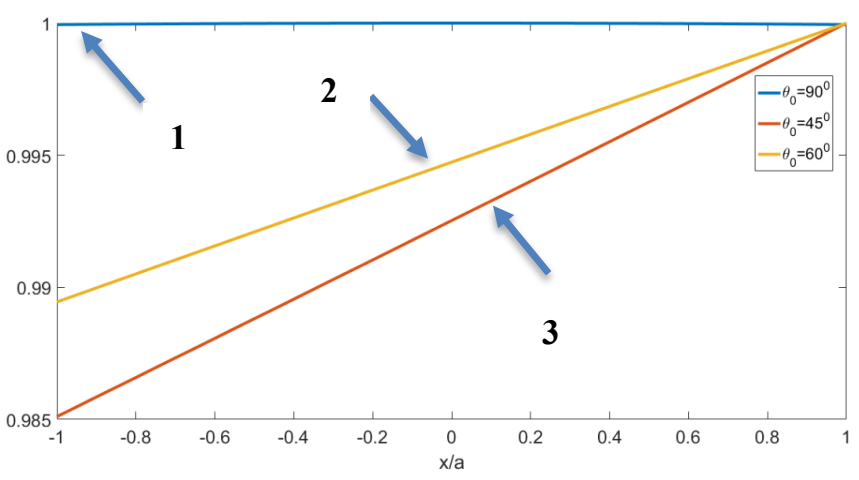

Figure 11: The normalized Fractional Current Density $\left|\tilde{f}^{1-v}(\xi)\right|$ for $\epsilon=\pi$ and $\rho_{0}=30$ 


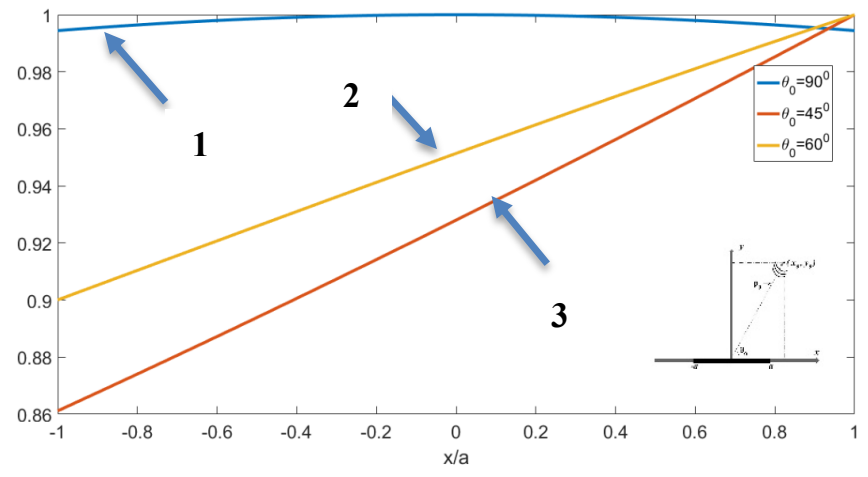

Figure 12: The normalized Fractional Current Density $\left|\tilde{f}^{1-v}(\xi)\right|$ for $\epsilon=3 \pi$ and $\rho_{0}=3$

\section{Conclusion}

In this study, the scattering analysis of a cylindrical wave from an impedance strip by using the fractional derivative approach (FDM) is studied. The problem of diffraction of the cylindrical wave by a strip with $\mathrm{FBC}$ has been formulated and for fractional order $v, 0.5$, the analytical expression of the field is found. FBC is characterized by the value of the fractional order $v$ between 0 and 1 . The impedance of the strip is found for the specific angle with the special assumption. When the fractional order $v$ equals to 0.5 , the impedance of the strip becomes $-i$ for the normal incidence (valid for large value $k \rho_{0}$ ). If there was a surface with the impedance $-i$, the properties of scattering of the cylindrical wave from the two-dimensional strip would be the same as the study done here. Such a surface has a very special characteristic in the sense of the radiation and the current distribution. As shown in the paper, the current distribution for $v, 0.5$ is very smooth. Furthermore, there are resonances for the specific angles of the incidence depending on the frequency parameter $\epsilon$ given in (8) as seen in Bi-static RCS results whereas such resonances are not observed in PEC or PMC structures [9]. Previously, the monostatic radar cross section, the far-field expressions and the near field expression for $y>0$ were studied. Here, especially, the current distribution for specific angles, bistatic radar cross section and the near field distribution for $\mathrm{y}<0$ and $\mathrm{y}>0$ are investigated. This method can be applied for different geometries such as double strips as a resonator, two strips with an arbitrary angle and the distance between each other. Introducing the fractional derivative method can lead to a promising technique in the modeling of scattering from complicated surfaces when the fractional order is defined from physical parameters $[6,9]$.

\section{Acknowledgements}

We would like to thank Hulusi Açıkgöz and Fatih Dikmen for their support.

\section{References}

[1] Bateman, H. \& Erdelyi, A. (1953). Higher Transcendental Functions, Volume 2, McGraw-Hill, New York

[2] Oldham, K.B. \& Spanier, J. (1974). The Fractional Calculus: Integrations and Differentiations of Arbitrary Order, Academic Press, New York

[3] Engheta, N. (2000). Fractional Paradigm in Electromagnetic Theory, a chapter in IEEE Press, chapter 12, pp.523-553

[4] Hilfer, R. (1999). Applications of Fractional Calculus in Physics, World Scientific Publishing, ISBN 981-0234-57-0, Singapore

[5] Veliyev, E. I., Karacuha, K., Karacuha, E., \& Dur, O. (2018). The Use of the Fractional Derivatives Approach to Solve the Problem of Diffraction of a Cylindrical Wave on an Impedance Strip. Progress In Electromagnetics Research, 77, 19-25.

[6] E. I. Veliev, K. Karaçuha, E. Karaçuha," Scattering of a Cylindrical Wave from an Impedance Strip by Using the Method of Fractional Derivatives" XXIIIrd International Seminar/Workshop on Direct and Inverse Problems of Electromagnetic and Acoustic Wave Theory (DIPED),Tbilisi, Georgia, September 24-27, 2018

[7] Balanis, C. A., Advanced Engineering Electromagnetic, Wiley, 1989.

[8] Veliev E. and N. Engheta. "Generalization of Green's Theorem with Fractional Differintegration". 2003 IEEE AP-S International Symposium \& USNC/URSI National Radio Science Meeting, 2003

[9] E. I. Veliev, M. V. Ivakhnychenko, ve T. M. Ahmedov, "Fractional Boundary Conditions In Plane Waves Diffraction On A Strip," Progress In Electromagnetics Research, vol. 79, sf. 443462, 2008.

[10] M. V. Ivakhnychenko, E. I. Veliev, ve T. M. Ahmedov, "Scattering Properties Of The Strip With Fractional Boundary Conditions And Comparison With The Impedance Strip," Progress In Electromagnetics Research C, vol. 2, sf. 189-205, 2008.

[11] Honl, H., A.; Maue, W. \& Westpfahl, K. (1961). Theorie der Beugung, Springer-Verlag, Berlin

[12] K. Karaçuha, "Kesirli Türev Yöntemiyle Silindirik Dalganın Empedans Şeridinden Saçılması," in IX. URSI-TÜRKIYE'2018 BİLIMSEL KONGRESI, September 2018 (in Turkish) 